\title{
Caso clínico en el Hospital Nacional Arzobispo Loayza.
}

Editor de Sección: Dr. Enrique Cipriani Thorne

\section{Caso clínico:}

Paciente mujer de 47 años, natural y procedente de Lima, con antecedente de contacto tuberculoso tres años antes del ingreso (inquilino). En abril del 2002 presenta diarrea sin moco, sin sangre, 3 cámaras por día, intermitente (3-4 días a la semana), que duró aproximadamente 1 mes y cedió espontáneamente, además presentó edema e incremento de volumen abdominal, tos seca, disnea, pérdida de peso no cuantificada, por lo que acudió al Hospital Nacional Arzobispo Loayza en julio del 2002, donde se le hace el diagnóstico de TBC entero peritoneal y pleural, sale de alta con tratamiento con cuatro drogas. La paciente no mejora, presenta dolor pleurítico, disnea y tos, por lo que es nuevamente hospitalizada en agosto del 2002.

Al examen físico: Presión arterial: 105/60 FC: 94 x' FR: 23 x' Temperatura: $37.7^{\circ} \mathrm{C}$, Peso: $46 \mathrm{k}$ Talla: $1.54 \mathrm{~m}$. Paciente en mal estado de nutrición, piel tibia, seca, edema tercio inferior de piernas, cuello: ganglios cervicales y supraclaviculares bilaterales menores de 1 $\mathrm{cm}$., móviles, renitentes, no dolorosas. Pulmones: Amplexación disminuida, matidez y abolición del murmullo vesicular en $2 / 3$ inferiores de hemitórax derecho. Corazón: ruidos cardiacos rítmicos, de buena intensidad, no soplos. Abdomen: hígado a $2 \mathrm{~cm}$. del reborde costal, longitud hepática $13 \mathrm{~cm}$. Genitourinario: puño percusión lumbar negativa. Neurológico: no alteraciones.

\section{Exámenes auxiliares:}

Primera hospitalización (17/07/02 - 06/08/02): Hematocrito: $32 \%$, frotis de sangre: hipocromía + , anisocitosis ++, microcitosis + . Plaquetas 520,000, Leucocitos 5100 (Abastonados 4, Segmentados 66, Eosinofilos 2, Monocitos 4, Linfocitos 19). Parasitológico seriado y reacción inflamatoria en heces: negativos. Proteínas Totales: 6.05, Albúmina: 1.8, Globulinas: 4.25. Tiempo de Protombina: 13.5 s, INR: 1.18. Glucosa: $67 \mathrm{mg} / \mathrm{dl}$. Creatinina: $0.69 \mathrm{mg} / \mathrm{dl}$. Examen de orina: Densidad 1010, Proteínas: 30mg/dl, Leucocitos 18-20 x campo, algunos aglutinados y degenerados, cilindros granulosos. Urocultivo: negativo. Proteinuria 24 horas: 0.518 gramos. AgHBs: No reactivo. PPD $3 \mathrm{~mm}$. Examen de liquido ascítico y pleural (tabla $\left.\mathrm{N}^{\mathrm{o}} 1\right)$.

Laparoscopía Abdominal: Ligamento redondo, peritoneo parietal y visceral con pequeños gránulos blanco nacarados diseminados. Lóbulo izquierdo del hígado, con peritoneo conteniendo gránulos. Líquido peritoneal amarillo. No se realizó biopsia.

Segunda hospitalización: Proteínas totales: $7 \mathrm{gr} / \mathrm{dl}$, Albúmina: $2.2 \mathrm{gr} / \mathrm{dl}$, Sedimento de orina: Leucocitos 2-5, Hematíes 4-8, cilindros hialinos 1+, gérmenes 1+, PPD: $3 \mathrm{~mm}$, Proteinuria en 24 horas: 1.36 gr. Creatinina: $0.68 \mathrm{mg} / \mathrm{dl}$. Anticuerpos antinucleares: negativo, Anti DNA doble cadena: negativo, HIV: negativo. Complemento 3: $211.3 \mathrm{mg} / \mathrm{dl}$. Líquido pleural (tabla $\mathrm{N}^{\circ} 1$ ).

Ecografía Renal: Riñón derecho: Bordes uniformes, Diámetro longitudinal: $102 \mathrm{~mm}$, parénquima renal homogéneo, $20 \mathrm{~mm}$. de espesor, seno renal normal. Riñón izquierdo: Bordes uniformes, Diámetro longitudinal: $105 \mathrm{~mm}$, parénquima renal homogéneo, 22 $\mathrm{mm}$. de espesor, seno renal normal.

\footnotetext{
$*$ Jefe del Servicio de Nefrología "Carlos Monge Cassinelli”, Hospital Nacional Arzobispo Loayza Profesor Principal Universidad Peruana Cayetano Heredia. Jefe del Departamento de Patología del Hospital Guillermo Almenara Yrigoyen.
} 
Tabla $\mathbf{N}^{\circ}$ 1. Examen de líquido ascítico y pleural.

\begin{tabular}{|l|c|c|}
\hline \multicolumn{1}{|c|}{ Prueba } & Julio & Agosto \\
\hline Liquido Ascitico & & \\
\hline Proteínas & 3.96 & \\
\hline Albúmina & 1.08 & \\
\hline Células & 45 & \\
\hline LMN & $100 \%$ & \\
\hline ADA & 40.7 & \\
\hline BK & Negativo & \\
\hline Liquido Pleural & & \\
\hline Proteínas & 4.25 & 4.98 \\
\hline Albúmina & 1.02 & 1.7 \\
\hline Células & 12 & 12 \\
\hline LMN & $100 \%$ & $100 \%$ \\
\hline ADA & 42 & 23 \\
\hline
\end{tabular}

Se realizaron biopsias de: ganglio cervical, cuyo resultado fue: Necrosis caseosa con reacción granulomatosa de células epitelioides periféricas. Pleural, siendo informada como: pleuritis crónica granulomatosa de tipo TBC. La paciente fue sometida a biopsia renal diagnostica.

\section{DISCUSIÓN}

\section{Dr. Abdías Hurtado Aréstegui*}

El caso es el de una paciente con diagnostico definido de Tuberculosis (TBC) extrapulmonar, que compromete pleura, peritoneo y ganglios, en la cual se demuestra un estado anérgico por un PPD negativo (2mm) y la presencia de compromiso renal expresado por una proteinuria significativa.

La TBC es un problema de salud pública tanto en países en vías de desarrollo como en los desarrollados. En el mundo existen 16.2 millones de casos de TBC y cada año aparecen alrededor de 7.9 millones de casos nuevos. El $95 \%$ de estos casos ocurre en países en vías de desarrollo (1). En el Perú los principales indicadores epidemiológicos demuestran un descenso de la TBC, aunque estos siguen siendo altos comparados con otros países, así para 1999 se diagnosticaron 41,730 personas con TBC, la tasa de morbilidad fue de 165.4 por 1000,000 habitantes, los casos nuevos 24511 , la tasa de incidencia de 97.1 por 1000,000 habitantes $(1,2)$.

La TBC es causada por Mycobacterium tuberculosis y es una enfermedad multisistemica, siendo el pulmón el órgano mas afectado. El compromiso extrapulmonar se observa entre el 10 al $17 \%$ de los pacientes y se origina por diseminación linfo-hematogena de un foco primario (menos frecuentemente de un foco pulmonar secundario o una localización extrapulmonar), e implica algún grado de compromiso de la inmunidad (3-5). La Patogénesis de la TBC esta determinada por el ingreso del bacilo tuberculoso por vía aérea al pulmón donde forma un complejo primario, la infección inicial ocurre generalmente en ausencia de linfocitos $\mathrm{T}$ que puedan reconocer los antígenos del Mycobacterium tuberculosis lo que permite al organismo multiplicarse y diseminarse tempranamente a otros órganos por vía linfática hacia ganglios regionales y hematógena hacia cualquier órgano. Dos tipos a 4 semanas después de la infección, se desarrollan dos respuesta inmunológica: una reacción de hipersensibilidad retardada en reacción a antígenos bacilares y otra mediada por células que resulta en la activación de macrófagos capaces de digerir al bacilo tuberculoso y de presentar antígenos a los linfocitos $\mathrm{T}(6$, 7). Los linfocitos inducen protección a través de la producción de linfoquinas, su proliferación es crucial en la defensa del organismo contra M. tuberculosis. Por ello los defectos cuantitativos y cualitativos de los linfocitos $T$ $\mathrm{CD}^{+}$explican la incapacidad de individuos infectados con HIV para contener la proliferación de la micobacteria. La respuesta inmunitaria es capaz de inhibir el crecimiento bacilar y la infección es controlada en el complejo primario en un 90 a $95 \%$ de pacientes, generando un estado quiescente aún con presencia del bacilo.

La TBC renal es una forma frecuente de compromiso extrapulmonar, la infección compromete principalmente la médula renal, los pacientes afectados pueden cursar asintomáticos hasta en un $25 \%$ ó presentar piuria aséptica, hematuria, infecciones urinarias recurrentes, en estos casos

Figura $\mathbf{N}^{\circ} 1$. Patogenesis de la TBC renal
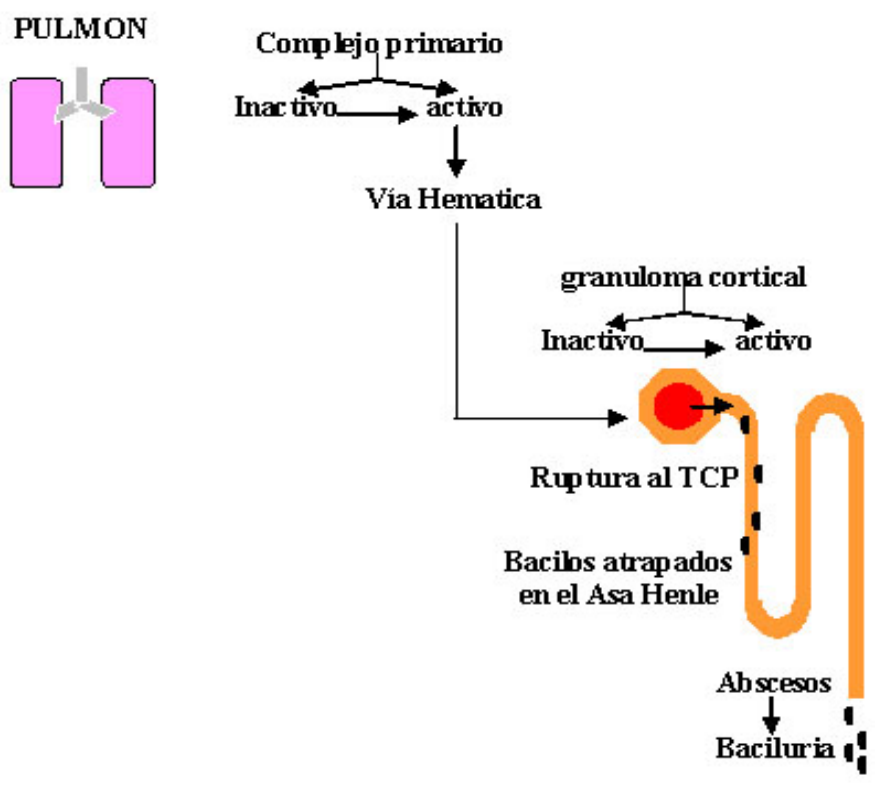
el diagnóstico se realiza por la demostración del bacilo tuberculoso en cultivos seriados de orina (5). El daño a nivel renal se genera en forma similar al que ocurre en el pulmón, el bacilo se localiza inicialmente en el glomérulo (favorecido por la adecuada oxigenación, alto flujo de sangre e incremento de viscosidad en la arteriola eferente), las células mesangiales secretan linfoquinas, induciendo la infiltración de macrófagos y formación de granulomas corticales (4), estos pueden ir a la cicatrización o romperse dentro del túbulo proximal, los bacilos son atrapados en el asa de Henle donde se multiplican (debido a la disminución de la respuesta inmune ocasionada por la hipertonicidad, pobre flujo sanguíneo, alta concentración de amonio), formándose granulomas medulares.

Consecutivamente los macrófagos del granuloma atraen fibroblastos y estimulan la secreción del factor de necrosis tumoral, esto determina la formación de tuberculomas y necrosis caseosa; luego los focos caseosos se rompen hacia el sistema colector formando cavernas (figura $\mathrm{N}^{\circ} 1$ ). En la paciente no tenemos información acerca de cultivo de BK en orina que es la forma como debe hacerse el diagnostico y tenemos una ecografía renal que no muestra anormalidades estructurales.

Otra forma de presentación de la TBC a nivel renal, es como síndrome nefrótico, en estos casos la TBC se comporta como un estimulo inflamatorio crónico que determina la deposición de amiloide en el riñón; en el Perú la amiloidosis se encuentra en el $9.5 \%$ de las biopsias renales (7), este diagnóstico se aleja en la paciente debido a que la proteinuria no es de rango nefrótico.

Se ha descrito diferentes tipos de glomerulonefritis asociadas a TBC (8-15); en los que la expresión clínica ha sido proteinuria, síndrome nefrótico o insuficiencia renal. En estos casos se ha propuesto que el daño glomerular es producido por un mecanismo inmunológico iniciado por la TBC, sin embargo no se ha podido demostrar ningún antígeno tuberculoso a nivel del glomérulo. La TBC y otras enfermedades activan el sistema inmunológico, la respuesta de este sistema determina diferentes expresiones clínicas. La comprensión de la respuesta inmune ha cambiado gracias al conocimiento de que los linfocitos $\mathrm{T} C D 4^{+}$ se diferencian funcionalmente en dos subgrupos: $\mathrm{T}$ helper 1(Th1) o T helper 2 (Th2) (figura $\mathrm{N}^{\circ} 2$ ), esto ha ayudado a entender mejor los diferentes patrones inmunológicos y clínicos de las enfermedades (16 - 18).

Por ejemplo, los pacientes infectados con Mycobaterium leprae pueden tener una presentación clínica como lepra tuberculoide, una enfermedad mediada por activación de linfocitos Th1 de característica limitada, con una fuerte respuesta de
Tabla N². Glomerulonefritis Membranosa: Causas

\begin{tabular}{|l|l|}
\hline Primaria & \\
\hline Secundaria: & \\
\hline Infecciones & Hepatitis B, C \\
& Sífilis \\
& Esquistosomiasis \\
\hline Enfermedades & Lupus Eritematoso Sistemico \\
Autoinmunes & Artritis Reumatoidea \\
& Sjogren \\
\hline Neoplasias & Tumores solidos (Carcinomas) \\
\hline Drogas & Sales de Oro \\
& Penicilamina \\
& Captopril (raro) \\
& AINEs (Diclofenaco, raro) \\
\hline
\end{tabular}

inmunidad celular y formación de granulomas; o presentarse como lepra lepromatosa enfermedad más extensa con una fuerte producción de anticuerpos mediada por activación de linfocitos Th2. En la TBC la respuesta inmune predominante es del subgrupo Th1: mediada por células e hipersensibilidad retardada. Sin embargo, los pacientes que desarrollan TBC extrapulmonar o TBC miliar, presentan una disminución de la inmunidad, una fuerte respuesta de anticuerpos característica de la activación de linfocitos Th2 (18).

En el caso de nuestra paciente la evidencia de enfermedad extrapulmonar (ganglionar, pleural), PPD negativo, hipergamaglobulinemia, sugiere activación Th2, por lo que se puede plantear que el tipo de glomerulonefritis que se encontraría seria lesiones mínimas o GN membranosa (característica de enfermedad por complejos inmunes).

\section{Grafico $\mathbf{N}^{\circ}$ 2. Diferenciación Funcional de Linfocitos T Helper en SubGruposS Th1 y Th2 y Cuadros}

\section{Clínicos Asociados}

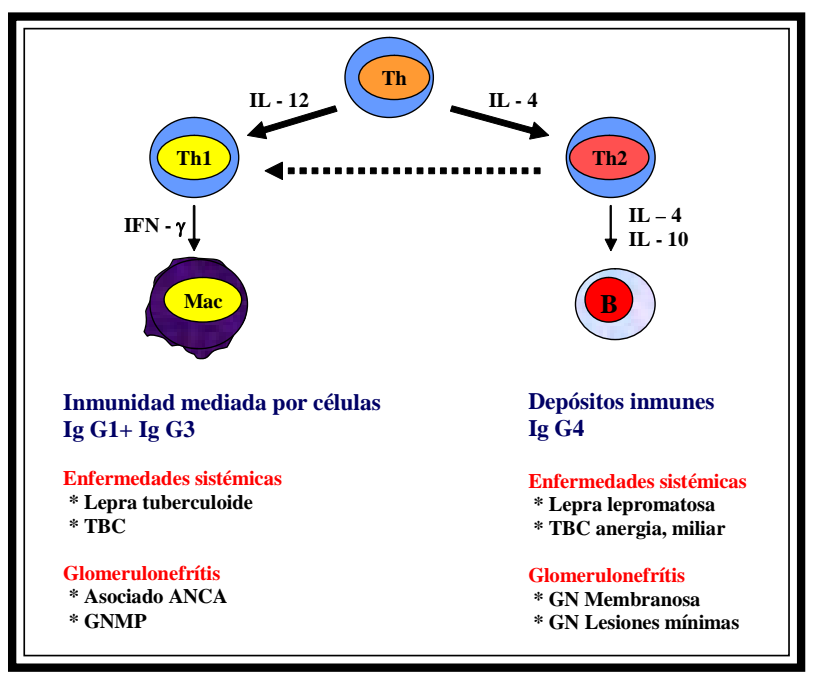


Foto $\mathbf{N}^{\circ} 1$.

Biopsia Renal: Glomerulonefritis membranosa (Hematoxilina eosina)

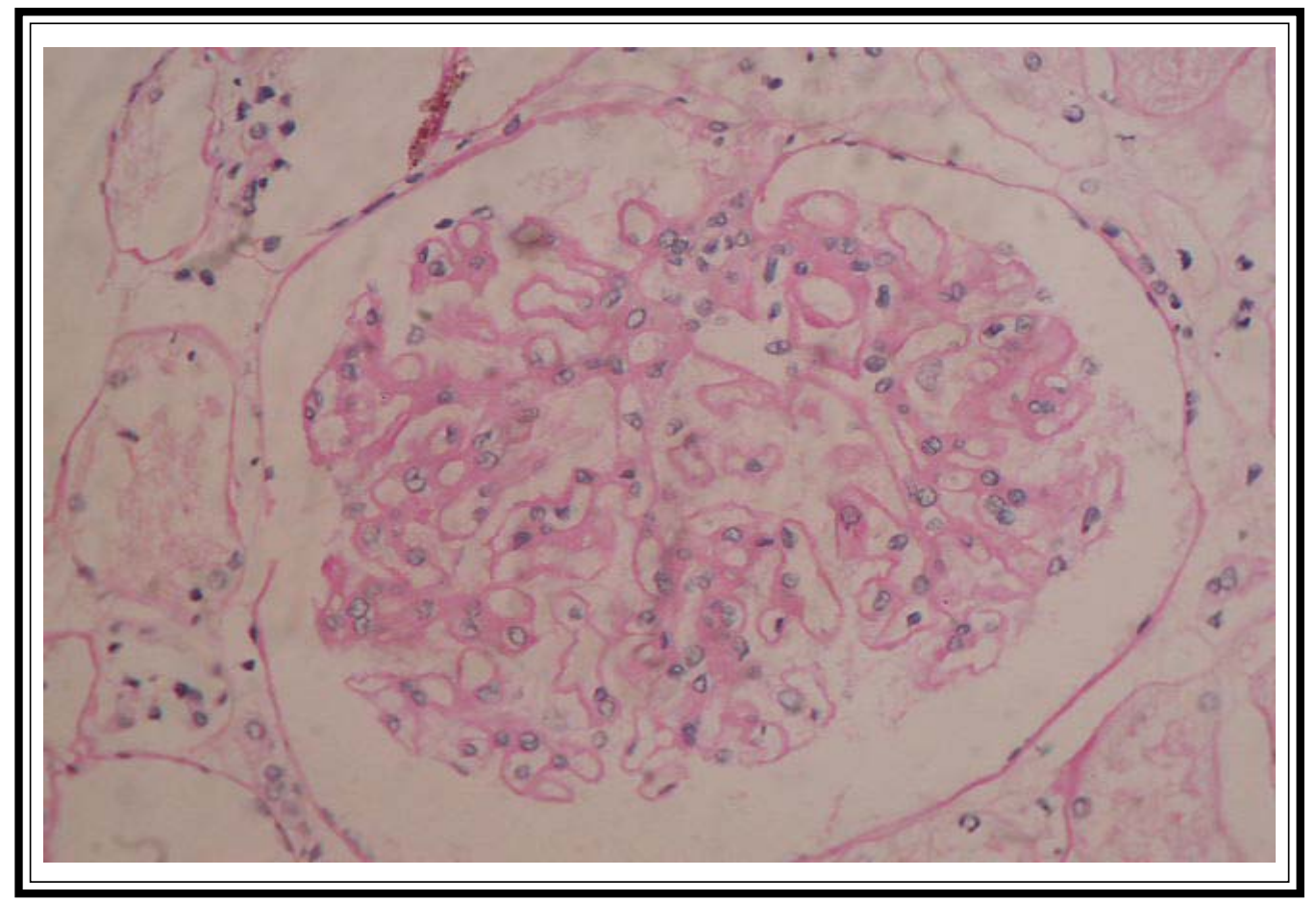

Foto $\mathbf{N}^{\circ} 2$.

Biopsia Renal: Glomerulonefrítis membranosa (Inmunofluorescencia)

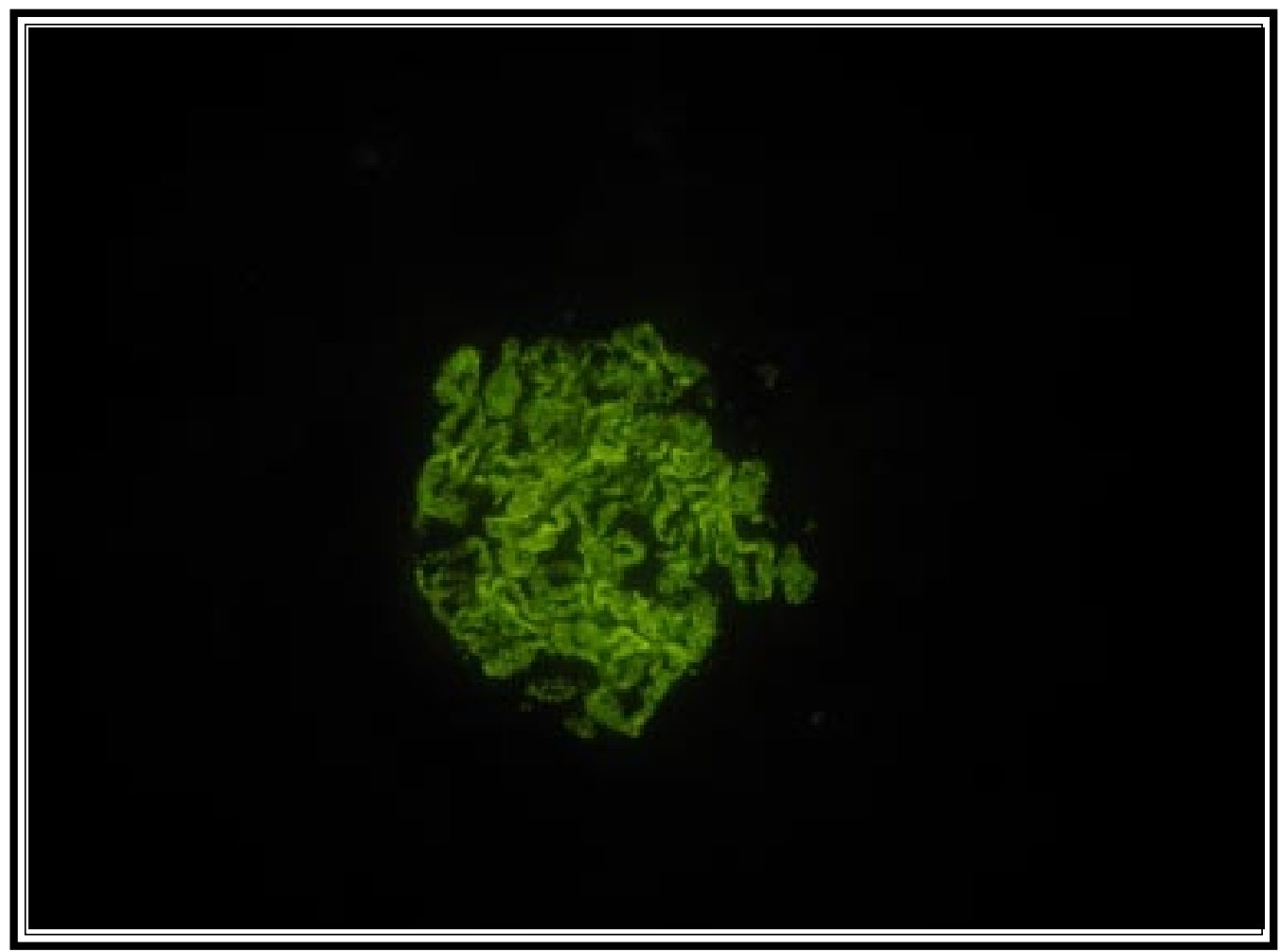


Diagnostico clínico:

TBC Extrapulmonar

Glomerulonefritis secundaria a TBC:

GN membranosa o GN lesiones mínimas

\section{Dra. Carmen Asato Higa **}

La biopsia recibida fue adecuada; todos los glomérulos muestran engrosamiento global de sus asas capilares, con presencia de espículas en la coloración argéntica y sin mayor alteración del mesangio; hallazgos característicos de la glomerulopatía extramembranosa (Foto $\mathrm{N}^{\circ} 1$ ), lo cual es corroborado en el estudio inmunofluorescente, en el que se evidencia depósitos granulares con anti IgG y anti C3 humano a lo largo de todas lasa asas capilares (Foto $\mathrm{N}^{\circ} 2$ ). Los túbulos muestran degeneración turbia de su citoplasma. No se encontró granulomas, ni nefritis intersticial. En la biopsia no se hallo signos de progresión de enfermedad renal.

La glomerulonefritis membranosa puede ser Primaria: en estos casos no se encuentra un factor que explique la glomerulonefritis o ser secundaria a diversas enfermedades, (Tabla $\mathrm{N}^{\circ} 2$ ), siendo las mas frecuentes: Infecciones (Hepatitis B ó C, Sífilis), Enfermedades inmunologicas (Lupus eritematoso sistémico), Drogas (Sales de oro, Penicilamina) y Neoplasias. En esta paciente se descartó las causas anteriormente mencionadas, se tiene evidencia de tuberculosis extrapulmonar a nivel pleural y ganglionar, lo que sugiere que la lesión renal es secundaria a la infección tuberculosa.

\section{Diagnóstico patológico:}

Glomerulonefritis membranosa secundaria a TBC.

\section{REFERENCIAS BIBLIOGRAFICAS}

1. Tuberculosis en el Perú: Informe 1999.- Lima: Dirección general de la salud de las personas 2000.

2. Ministerio de Salud-Proyecto VIGIA. Tuberculosis en el Perú. Informe 2000.

3. Haas D, Des Prez R, Mycobacterium tuberculosis. In Mandell, Douglas and Bennett's principles and practice of infectious diseases, 1995. pp:2213-2243.

4. Visweswaran K, Bath S. Tuberculosis of the urinary tract.
Incomprehensive Clinical Nephrology, Johnson R, Feehally J. 2000.

5. Rubin R, Cotran R, Tolkoff-Rubin N. Urinary tract infection, pyelonephritis, and reflux Nephropaty, In Brenner and Rector's the Kidney $5^{\text {Th }}$ Ed. 1996. pp:1638-39.

6. Patrucco Raúl, Vargas M., Torres I. Estudio de los Parámetros Inmunológicos en pacientes portadores de Tuberculosis activa. Diagnóstico 1983;12:132-137.

7. Hurtado A, Escudero E, Stromquist C, Urcia J, Hurtado ME, Gretch D, Watts D, Russell K, Asato C, Johnson R. Distinct patterns of glomerular disease in Lima, Perú. Clin Nephrol 2000;53:325-332.

8. Hubner J, Zavala R, Garcia U, Sanchez L. Compromiso glomerular en tuberculosis. Diagnostico 1983; 12: 189-193.

9. Schribman H, Eastwood B, Uff J. Immune complex nephritis complicating miliary tuberculosis. Br Med $\mathbf{J}$ 1983;287:1593-1594.

10. Meyrier A, Valensi P, Sebaoun J. Mesangio-capillary glomerulonephritis and the nephrotic syndrome in the course of disseminated tuberculosis. Nephron 1988;49: 341-342.

11. O'Brien AA, Kelly P, Gaffney EF, Clancy L, Keogh JA. Immune complex glomerulonephritis secondary to tuberculosis. Ir J Med Sci 1990;159:187.

11. Rodriguez-Garcia JL, Fraile G, Mampaso F, Teruel JL. Pulmonary tuberculosis associated with membranous nephropathy. Nephron 1990;55:218-9.

12. Villar I, Hernandez E, Cozzi J, Paletta C, Mathurin S. Immune complex glomerulonephritis associated with pulmonary tuberculosis. Medicina (B Aires) 1994;54 :237-40.

13. Sopena B, Sobrado J, Javier Perez A, Oliver J, Courel M, Palomares L, Gonzalez L. Rapidly progressive glomerulonephritis and pulmonary tuberculosis. Nephron 1991;57:251-2.

14. Pecchini F, Bufano G, Ghiringhelli Membranoproliferative glomerulonephritis secondary to tuberculosis. Clin Nephrol 1997 ;47:63-4.

15. Matsuzawa N, Nakabayashi K, Nagasawa T, Nakamoto Y. Nephrotic IgA nephropathy associated with disseminated tuberculosis. Clin Nephrol 2002; 57:63-8.

16. Holdsworth S., Kitching A., Tipping P. Th1 and Th2 helpar cell subsets affect paterns of injury and outcomes in glomerulonephritis. Kidney International 1999; 55:1198216.

17.Thomas M. Daniel, Oxtoby Margaret, Pinto Enrique, Moreno Edgar. The Inmune spectrum in patients with pulmonary tuberculosis. Am Rev Dis 1981; 123:558-559.

18. Montiel M, Rivera S, Hernández R, Hassanhi M, Vargas F, Núñez J. Respuesta inmunitaria y anergia, estudios en pacientes tuberculosos del hospital universitario de Maracaibo, Venezuela. Acta Científica Venezolana 2002;53:36-43. 